\title{
Lockdown: Abandon targets or leave future of dental services at risk
}

The British Dental Association has urged the government to abandon imposed NHS targets that will be impossible to achieve under the new national lockdown, and which risk putting hundreds of practices out of business.

The union had refused to sign up to plans that took effect on New Year's Day which will see practices face steep financial penalties if they fail to hit $45 \%$ of their pre-pandemic NHS activity targets from 1 January to 1 April 2021. In an open letter it has called on Health Secretary Matt Hancock to drop the activity measures with immediate effect.

The BDA believes that the latest government restrictions have a major impact on patients' willingness to seek care, whilst the NHS targets will force dentists to prioritise routine check-ups for the 'worriedwell' over a time-consuming urgent backlog.

Two fifths (41\%) of practices currently operating below $36 \%$ of their targets face a 'cliff edge' and the potentially ruinous return of the majority of their NHS funding for the period 1 January to 1 April 2021.

NHS practices have been struggling to see more than a fraction of their pre-pandemic patient numbers owing to tight social distancing and decontamination restrictions, with over 19 million appointments delayed since lockdown. The Welsh Government has set no targets and set aside dedicated funding for ventilation equipment that can help practices rebuild capacity. Scottish and Northern Irish authorities have set targets at less than half the planned level for England.

Dave Cottam, Immediate Past Chair of the British Dental Association's General
Dental Practice Committee said: 'As the nation returns to lockdown, the targets imposed by Government on NHS dentistry are now impossible to justify.

'Dentistry remains open and safe, but patients will understandably now have real concerns about attending.

'This policy was already set to push NHS practices to the brink. Without urgent change, dental services across this country face decimation, risking patient care for years to come.

Meanwhile the Faculty of General Dental Practice UK (FGDP), College of General Dentistry (CGDent) and the Faculty of Dental Surgery of the Royal College of Surgeons of England have issued a joint message that safety must take clear priority over dental activity levels during the latest national coronavirus lockdown.

The leading dental organisations have also highlighted the continued applicability of their guidance, and reiterated the need to consider the prevalence of COVID-19, and local rates of infection, when carrying out risk assessments.

Ian Mills, Dean of the FGDP, said: 'Many patients have been unable to access dental care for over a year, with their dental problems getting steadily worse, and oral health inequalities exacerbated by coronavirus restrictions. The dental profession is acutely aware of the present backlog of unmet dental need, which requires treatment as soon as possible. However, the recent imposition of UDA based targets in England to remedy this, just as the pandemic becomes worse than it has ever been, is at odds with the
Government's message to 'stay at home to protect the NHS and save lives'.

'The dental profession has worked assiduously to create a safe environment for dental treatment to be delivered, but there are justifiable concerns that significant increases in patient throughput, in order to meet UDA targets, could potentially compromise staff and patient safety. The UDA was an ineffective measure of activity before the COVID pandemic, and we consider it even less appropriate now. Circumstances will vary by practice and by patient, but the priority should be on safety, the treatment of those with acute needs, and essential maintenance of oral health.'

Matthew Garrett, Dean of the Faculty of Dental Surgery of RCS England, added: 'Positive tests for COVID-19 and COVID-related deaths are at record levels, emergency departments and intensive care units are under severe pressure, and hospital staff are stretched like never before. Dental teams have robust infection prevention and control protocols in place, which can reduce the risk of transmission if rigorously applied, but we cannot eliminate it altogether, not least given the risks inherent in patients travelling to appointments.

'Encouraging greater throughput at the present time may not be in the best interests of patients with routine oral health needs, nor does it support the NHS and the national coronavirus response. However, the public should be reassured that dental practices are safe places to visit if they need to access urgent care.

\section{Scotland: Update on PPE, ventilation and private practice guidance}

NHS National Services Scotland (NSS) have confirmed that the current arrangements for supplying dental practices with a limited amount of free PPE will be extended to the end of June. This should provide some reassurance about the continued supply of PPE to practices. It comes after our extensive work with NSS on PPE supplies for dentists, including reported problems with local communication and provision.

Regarding ventilation, the Scottish Government has admitted that ventilation is a fundamental part of the 'COVID-19 armoury' - despite recent suggestions the new Health Minister is unwilling to set aside dedicated funding.

David McColl, Chair of the Scottish Dental Practice Committee, said: 'Dental practices still face tight restrictions that have radically reduced the number of patients we can treat. At the very least, dentists need clear guidance from the Scottish Government about what they should be doing about ventilation.'
The status of, and restrictions on, wholly private practices were also confirmed. The National Clinical Director and Chief Dental Officer issued a joint letter to confirm that dentistry is essential healthcare and that wholly independent or private practices can deliver a comparable service to that available through the NHS This letter also clarified that, cosmetic or aesthetic procedures are not regarded as essential and must not be provided by any practice in Level 4 areas. 